resins are recovered from oil residues, soaps are made stable in colour and perfume, waste molasses is made into tiles and insulating materials, broken rice grains of little or no value are changed into colloidal solutions and used for making synthetic rice grains, oils are purified so as to burn without smoking. Researches of a fundamental character are being made into the magnetic properties of atoms and molecules with the object of revealing the nature of chemical bonds and the control of chemical reactions. At the Tata Institute at Bangalore the experiments in the physics section on backward internal scattering of light in liquids and the effect of supersonic waves on refraction show a combination of high experimental skill and first-class apparatus. A demonstration of the structure of lustrous shells was beautiful and impressive.

Among other notes, one of the delegates was struck with the initiative shown in making apparatus, which otherwise would have been hard to come by in India, at the Central Meteorological

\section{The Aurora of}

$\mathrm{T}$ HE auroral display on the night of January 25-26 was remarkable not only for its brilliance but also for the wide area over which it was observed. Some time must elapse before it is possible to collect complete information, but it seems certain, from the newspaper reports already published, that it was seen over practically the whole of Europe, and as far south as Gibraltar and Sicily. The Times of January 27 states also that vivid auroral displays were seen over a large part of Canada on the nights of January 24-25 and 25-26.

Accounts of the phenomenon as observed in the British Isles differ considerably in points of detail. It is proposed to give here a few extracts from the most precise reports received either by NATURE or by the Meteorological Office. An account of the appearances observed at Harpenden, Herts, by Dr. B. A. Keen was given in last week's issue. Dr. Keen observed the display from 6.45 until after midnight. Three periods of brilliant display were noted, around $7.45,8.30$ and 9.45. The main features noted were red glows in the northwest and north-east, with a low green arc between during the early stages, green and white rays traversing a bright red glow in the north-north-east about 7.45, and rapidly fluctuating green streamers between north-west and north-east about 9.45 .

At Kew Observatory, detailed observations were made between 6.50 and 9.10. These observations show good general agreement with those made by Dr. Keen, except that a green coloration of the
Station for altitude observations in Agra. Another comments upon the fine work of the Hydrodynamic Research Station at Poona, where, beneath the great irrigation dam, large-scale experiments are possible and have resulted in numerous and various records covering the work of years and published by the Government of India, but yet to be incorporated, as they should be, in text-books on hydraulics.

Such are a few examples and it must be emphasized that they are only a few among many

of the widespread and practical interest in the advancement of science and its applications encountered by the delegation in India. If the delegation, through the contacts which its members have made, has succeeded on one hand in aiding that advancement in any manner, and should succeed, on the other hand, in broadening understanding at home of the achievements of scientific workers in India, it will have discharged its most important functions for the benefit of science in both countries.

\section{January $25-26$}

arcs and rays is not mentioned. Strong groups of coloured rays were observed after 7.35 , and at 7.51 these were spreading out from north-northeast to west-north-west reaching elevations of $5^{\circ}$ to $10^{\circ}$ above Polaris. At 7.57 the glow extended slightly beyond the zenith. A few minutes later, at 8.07, the display faded completely and no aurora was visible for several minutes. The later stages as observed at Kew were distinctly less brilliant, though there were some further appearances of arcs, patches and rays.

Mr. Charles Leaf sends details of observations made near Cambridge from 1.15 on January 26. He writes as follows:

"As seen at 1.15 G.M.T. there was a bright greenish glow behind banded cirrus cloud right across the northern horizon, while just to the north-west of the-zenith there were two parallel bands of delicate pinkish light. Short groups of rays frequently developed in these bands. The rays were paler than the bands themselves, and gave the impression that they were white rays showing through the bands. The pink coloration gradually spread across the zenith towards the south-east where it appeared as a darker red patch, due to the background not being illuminated. At the same time there appeared a few faint luminous marks to the north-east, again quite close to the zenith. By 1.35 all that remained was a much fainter glow to the north and north-west, and this persisted until 2.30 , when I went to bed."

A letter received in the Meteorological Office 
from Mr. J. M. Brierley, of Rodwell, South Petherton, Somerset, describes the phenomena as seen on a motor-car journey from Honiton to Ilminster. Here a rosy glow was observed in the north-north-west as early as 6.0 o'clock. By 6.30 the glow had increased greatly in size and brilliance, and numerous pale green streamers were observed. Until 8.05 the main features were a general display of rose-coloured light above a greenish arch extending almost from west to east, and reaching almost to the zenith. A period of maximum brilliance occurred at 8.05 ; the greenish arch was then at an elevation of about $45^{\circ}$, and above this was a rose-coloured arch nearly $90^{\circ}$ broad. Numerous bright streamers appeared along the whole greenish arch and converged to a point between Aldeboran and the Pleiades. The green arch persisted until 11 p.m., after which the display was terminated by cloud.

Mr. R. Moss, Wellington College, Berks, has sent us a copy of a detailed record of observations made by two pupils, M. Majendie and J. $H$. Gordon, at the College, between 6 p.m. and 11.30 p.m. The period of maximum activity and brilliance was noted to have occurred between 7.45 and 8.0 o'clock and a second very active period between 8.30 and 8.45. Observations of the characters and extensions of different parts of the display were made every few minutes, and notes were written down at once, so that the record has far greater value than the general accounts of the display usually regarded as sufficient. A copy of the notes has been sent to the auroral branch of the British Astronomical Association, and $\mathrm{Mr}$. Moss will be pleased to supply further information concerning the observations to anyone especially interested in them.

In a leading article in The Times of January 27 , reference was made to the belief, going back to the time of Tacitus, that the aurora gives forth sounds. It is of interest to record that an observer at Catcott, Bridgwater, Somerset, the Rev. Harold Buckton, states that he seemed to hear now and again faint clicks resembling the noises made by the sparking of an electric machine. He is positive that these sounds were not caused extraneously, and he was not in fact aware of the possibility of sounds in association with the aurora, until he saw the matter referred to in The Times. The modern view is that sound could not reach the earth's surface from an electrical discharge occurring at heights of sixty miles or more, but we include the observation in these notes for what it is worth.

The subject was referred to by Prof. A. S. Eve in a discourse delivered at the Royal Institution on February 7, 1936, and published in a supplement of Nature of May 16, 1936. Prof. Eve's view is that the aurora can make little or no noise, and that what is heard is of the nature of local brush discharge such as is frequently associated with electrical disturbances. An interesting letter upon the subject appeared in our issue of December 2, 1933, from F. T. Davies and B. W. Currie, of the Canadian Meteorological Office, Toronto.

\section{Solar and TerRestrial Relationships}

The great aurora and magnetic storm of January 25 last was a spectacular reminder of a solar-terrestrial relationship, the evidence for which has been slowly accumulated for nearly a hundred years. Although the connexion between magnetic storms and the aurora borealis (or australis) is not a simple one, yet there is in general a close association between the two phenomena. The observation of disturbances in the earth's magnetic field is a fairly straightforward matter, requiring, of course, a specially prepared magnetic observatory, but being independent of the weather. On the other hand, data of the aurora are disturbed by cloudy weather and the periodic occurrence of bright moonlight. As defined at Greenwich, a small magnetic storm is a disturbance in which the range in magnetic declination equals or exceeds $\frac{1}{2}^{\circ}$ or that in horizontal force or vertical force equals or exceeds $150 \gamma$; a great storm is one having double these ranges as minimum (see NATURE of January 29, p. 192).

The evidence for a solar connexion with magnetic storms and associated auroras may be briefly summarized as follows: (1) a general accordance between the frequency curves of sunspots (or some other suitable criterion of solar activity) in the well-known eleven-year cycle and similar curves of magnetic storms. (2) An observed tendency for the lesser magnetic storms to recur at intervals of about twenty-seven days, which is the time taken for the sun (more particularly the mean latitude of the sunspot zones, one north and the other south of the sun's equator) to rotate once on its axis with respect to the earth moving in its orbit around the sun. Auroras likewise show this same recurrence tendency in a period of twenty-seven days. (3) A statistical relationship existing between the occurrence of individual sunspots of great size and the occurrence of great magnetic storms and associated auroral displays. Specific cases of joint coincidences between great sunspots, great magnetic storms, and unusual auroral displays witnessed in the south of England include those of March 22-23, 1920 ; August 9-10, 1917 ; November 17, 1882 ; August 28 and September 2, 1859, the last being associated with the unique observation, made by Carrington and Hodgson, of a transitory bright spot on the sun seen in integrated light with an ordinary telescope. At the commencement of a great 
magnetic storm, the associated sunspot is found on the average about one day past the sun's central meridian. This asymmetry with respect to the central meridian is thought to be indicative of the time of travel required for the solar agency to travel from the sun to the earth. An informative feature, often shown by great magnetic storms, is their abrupt commencement at the same time (U. T.), so far as can be determined at widely separated magnetic observatories.

Apart from these special disturbances in the earth's magnetic field, illustrated by the recent aurora and magnetic storm, mention should be made of the high degree of accordance between curves of some solar index (say, smoothed mean daily areas of sunspots for each month) and curves similarly prepared which give the mean daily ranges of the earth's magnetic elements, $D, H$ and $V$, after the seasonal inequality has been eliminated. No other known terrestrial phenomenon gives such detailed accordance with the observed periodic curve of solar activity.

To revert to the coincidences shown statistically by individual sunspots of first magnitude and great magnetic storms, it should be noted that as we proceed to the lesser sunspots and lesser magnetic storms, the relationship becomes less and less evident, and one concludes that the solar disturbances primarily related to the magnetic storms and associated auroras do not always produce a visible marking on the sun such as a sunspot. This point is clearly brought out in "time-pattern" diagrams prepared by J. Bartels to show synchronous or nearly synchronous solar activity and magnetic activity on the earth. The occurrence of the recent aurora is also an anomaly. Between January 12 and 24 a very large sunspot crossed the sun's disk. Magnetic storms were recorded in Great Britain commencing on January 16 and 22, and there were reported auroras in Canada. On January 25, when the great auroral display was seen, the big sunspot had been carried out of view by the sun's rotation. A spectroscopic examination of the sun's disk naturally suggests itself, but so far no distinctive phenomena have been reported which could reasonably be associated with the aurora and magnetic storm.

Characteristic features of the aurora itself have been investigated by studying (1) typical forms, (2) geographical distribution (annual and diurnal frequencies), (3) height above the earth's surface, (4) the characteristic spectrum. A useful collection of auroral photographs enabling the observer to identify typical forms is given in a "Photographic Atlas of Auroral Forms" published in 1930 by the International Geodetic and Geophysical Union. As is well known, the zone of maximum auroral frequency roughly circles the earth's magnetic poles in magnetic latitude $67^{\circ}$ (the position of the north magnetic pole being in geographical longitude $96^{\circ} \mathrm{W}$. and latitude $70 \frac{1}{2}^{\circ} \mathrm{N}$., and that of the south magnetic pole in longitude $151 \frac{1}{2}^{\circ} \mathrm{E}$., and latitude $71 \frac{1}{2}^{\circ} \mathrm{S}$.). Thus for places in the same geographical latitude, Canada is, for instance, more favourably placed for observing polar auroral phenomena than is Great Britain.

The association of magnetic disturbance with the aurora was first remarked by Halley in 1716 ; but as already noted, the association is not a simple one; auroras have been observed in the absence of marked magnetic disturbance and vice versa. Near the auroral zone itself, the frequency data of auroras fail to show a marked peak corresponding with solar maximum such as is given by the frequency curves of magnetic storms and auroras recorded in lower magnetic latitudes. It has been suggested by Vegard that the generally increased magnetic disturbance near sunspot maximum is instrumental in conveying auroras from the auroral zone to lower latitudes, thus reducing the frequency in the auroral zone itself. This suggestion finds corroboration in the results of a comparison made, by A. Röstad, between the position of auroral displays observed in Trondhjem, Oslo and in Holland and the intensity of the simultaneous magnetic storms recorded at Potsdam. Theoretical and practical investigations by Birkeland and Størmer have shown that if the earth encounters a beam of electrified particles, then the particles will be deflected by the earth's general magnetic field into two zones (to be identified with the auroral zones) one in the northern and the other in the southern hemisphere. The nature of these streams of particles has been the subject of several more recent investigations by Størmer, Chapman, Lindemann, Milne, Vegard, and others. Although the precise nature of the streams is undetermined, it would seem that they must consist of electrified particles of considerable energy so as to penetrate the earth's atmosphere to the lower observed limit of the aurora (about $70 \mathrm{~km}$.). It will be noted that the deflected corpuscles are able to come down on the night side of the earth, the diurnal distribution of the aurora showing a pronounced maximum near midnight, reckoned with respect to the earth's magnetic axis.

The determination of the height of auroras has in recent years been made systematically by measuring the angular displacement of distinctive features of a particular aurora against the background of bright stars, as recorded on two simultaneous photographs taken at opposite ends of a long base line. Eventually it should be possible to decide whether the aurora ever comes down to very low levels as is sometimes reported. 
The average height of the lower limit is about $106 \mathrm{~km}$. ; there is greater scatter for the upper limit, which shows some dependence upon type and distribution of light intensity along the streamers. The near coincidence in height between the average lower level of the aurora and that of the $E$-layer explored by radio-echo observations would appear to be other than fortuitous.

The spectrum of the aurora long presented great difficulty, and although important advances have been made, knowledge of the origin of the spectrum is by no means complete. In 1925, the well-known prominent auroral green line at $5577 \mathrm{~A}$. (discovered by Angström in 1866) was proved by McLennan and Shrum in their laboratory in Toronto to be due to oxygen in a metastable state. The great intensity of this line is explained by Vegard as being due to oxygen excited to emit this radiation by means of collisions of the second kind with activated nitrogen. Besides the prominent green line, there are two lines in the red, at $6300 \mathrm{~A}$. and $6365 \mathrm{~A}$., both of which are thought to be due to oxygen. In addition, the visual spectrum is characterized by the negative band spectrum of nitrogen. A phenomenon of great interest, studied in particular by Lord Rayleigh, is the light of the night sky, the spectrum of which shows the same green radiation at $5577 \mathrm{~A}$. as does the aurora, although the negative band spectrum of nitrogen is missing. Babcock accurately derived the wavelength as $5577 \cdot 35 \pm 0.01 \mathrm{~A}$. It has been suggested by Rayleigh (Proc. Roy. Soc., A., 106, 135) that the light from the non-polar aurora (as represented by the night sky luminescence found generally distributed over the world) is of a phosphorescent nature, the luminosity being excited by the sun during the daytime and carried round by the earth's rotation. It is important to note the distinction between the non-polar aurora and the polar aurora as exemplified by the recent display.

In conclusion, mention may briefly be made of the work of solar observers in trying to identify the precise phenomenon on the sun responsible for the occurrence of magnetic storms and their associated auroras. Replicas of Dr. Hale's spectrohelioscope, perfected by him in 1926, have been systematically used by a number of observatories in searching for and recording bright chromospheric eruptions which might, it was thought, be related to perturbations in the earth's magnetic field. Although no definite progress can be claimed in obtaining observational evidence of corpuscular streams (the search for which had been urged by Chapman), a new relation has been brought to light in which the solar agency appears to be radiation in the ultra-violet. There is now little doubt that sudden bright chromospheric eruptions (usually near sunspots) visible in $H \alpha$ with the spectrohelioscope or on $\mathrm{Ca}^{+}$spectroheliograms produce an immediate (that is, within about the time taken for light to reach the earth from the sun) disturbance in the ionosphere over the sunlit hemisphere, apparently just below the $E$-layer. At the same time, relatively small magnetic perturbations may occur, the effect of which is recorded within a limited distance from the place where the sun is in the zenith at the time of the solar eruption. These small disturbances in the earth's magnetic field are, however, clearly to be distinguished from the magnetic storms and auroras considered above, which are essentially high lattitude phenomena.

\section{Obituary Notices}

\section{Prof. W. H. Pickering}

DROF. W. H. PICKERING died on January 21 at his home at Mandeville, Jamaica, at the age of seventy-nine years, after several months of failing health. He was a younger brother of E. C. Pickering, the director of the Harvard College Observatory from 1876 until 1920.

Born at Boston in 1858, Pickering graduated at the Massachusetts Institute of Technology in 1879, and became a lecturer there, and at the same time was an assistant professor at the Harvard College Observatory. His first astronomical observations were at the eclipse of 1878 , where he observed the radial polarization of the corona. For a time he was in charge of the Harvard College Observatory Station at Arequipa. Vol. 33 of the Harvard College Annals contains his researches on the determination of photographic stellar magnitudes, the Great Nebula in Orion and visual observations of the moon and planets.
In 1900 Pickering established the Harvard College Observatory at Mandeville, and while there published a lunar atlas from photographs taken with a 12 -inch lens of focal-length $135 \mathrm{ft}$. The disk of the moon is. divided into sixteen parts, and each part photographed under five different illuminations. In 1898 he discovered by photography a small satellite of Saturn at a distance of 8 million miles from the planet and revolving round it in a year and a half. This satellite, to which he gave the name Phobe, is remarkable in that it revolves in a direction contrary to the other eight satellites, a peculiarity which was later found in three faint satellites of Jupiter.

At Mandeville, Pickering continued his visual observations of the moon and planets. He observed Mars under good climatic conditions and was satisfied of the existence of the "Canals" and drew the more important ones in agreement with Lowell. $\mathrm{He}$ regarded them as belts of vegetation of widths up to 150 miles along assumed water courses. In 1921 he 